\title{
Editorial: Mitochondria and Endoplasmic Reticulum Dysfunction in Parkinson's Disease
}

\author{
Sandeep Kumar Barodia ${ }^{1 *}$, Krishnan Prabhakaran ${ }^{2}$, Smitha Karunakaran ${ }^{3}$, Vikas Mishra ${ }^{4}$ \\ and Victor Tapias ${ }^{5 *}$ \\ ${ }^{1}$ Center for Neurodegeneration and Experimental Therapeutics, Birmingham, AL, United States, ${ }^{2}$ Department of Biology, \\ Norfolk State University, Norfolk, VA, United States, ${ }^{3}$ Centre for Brain Research, Indian Institute of Science, Bangalore, India, \\ ${ }^{4}$ Department of Pharmaceutical Sciences, Basanaheb Bhirao Ambedkar University, Lucknow, India, ${ }^{5}$ Feil Family Brain and \\ Mind Research Institute, Weill Cornell Medicine, New York, NY, United States
}

Keywords: Parkinson's disease, mitochondria, endoplasmic reticulum, $\alpha$-synuclein, PINK1, parkin, oxidative stress, MAMs

\section{Editorial on the Research Topic}

\section{Mitochondria and Endoplasmic Reticulum Dysfunction in Parkinson's Disease}

Endoplasmic reticulum (ER) and mitochondria are distributed in close communication via a dynamic ER-calcium $\left(\mathrm{Ca}^{2+}\right)$ mitochondria interconnection and regulate a plethora of vital cellular functions, including $\mathrm{Ca}^{2+}$ homeostasis, mitochondrial transport and dynamics, bioenergetics, ER stress, apoptotic signaling, and inflammation (Erpapazoglou et al., 2017). Alteration in the ER-mitochondria communication adversely affects overall physiology of the

OPEN ACCESS

Edited and reviewed by: Wendy Noble,

King's College London, United Kingdom

*Correspondence: Sandeep Kumar Barodia fnusandeepkumar@uabmc.edu Victor Tapias

vit2013@med.cornell.edu

Specialty section:

This article was submitted to

Neurodegeneration,

a section of the journal

Frontiers in Neuroscience

Received: 05 September 2019

Accepted: 16 October 2019

Published: 08 November 2019

Citation:

Barodia SK, Prabhakaran K, Karunakaran S, Mishra $V$ and Tapias V (2019) Editorial: Mitochondria and Endoplasmic Reticulum Dysfunction in

Parkinson's Disease.

Front. Neurosci. 13:1171 doi: 10.3389/fnins.2019.01171 cell (Gómez-Suaga et al., 2018). ER-mitochondria communication is also involved in lipid transport, suggesting that lipidomic approach may be useful to study the potential mechanisms leading to impaired neuropeptidergic signaling (Valadas et al., 2018). Mitochondria-associated membranes (MAMs) are defined as specialized subdomains connecting ER and mitochondria in order to regulate physiological functions, maintain $\mathrm{Ca}^{2+}$ signaling and other vital cellular processes (Rodríguez-Arribas et al., 2017). Neurons are highly dependent on MAMs to exchange metabolites and signaling molecules between ER and mitochondria, suggesting that altered function of MAMs due to toxin insults such as rotenone and manganese could play a crucial role in the pathogenesis of neurodegenerative diseases, including Parkinson's disease (PD) (Krols et al., 2016; Harischandra et al.; Ramalingam et al.; Valdinocci et al.). Modifications in the communication between ER and mitochondria cause a reduction in mitochondrial $\mathrm{Ca}^{2+}$ homeostasis in several animal models of neurodegeneration, such as PD, an age-dependent neurodegenerative disorder characterized by the progressive loss of dopamine (DA)-producing neurons in the substantia nigra (Paillusson et al., 2016; Lee et al., 2018). Several cellular mechanisms have been identified to be involved in the DAergic neuronal death, including mitochondrial dysfunction, impaired bioenergetics, oxidative stress, autophagy and impaired intracellular $\mathrm{Ca}^{2+}$ homeostasis in patientderived cell models of PD (González-Casacuberta et al.; Segura-Aguilar). However, mechanisms underlying how organelle crosstalk (especially between mitochondria and ER) could affect the progression of pathogenesis in PD still remain unknown. ER stress activates unfolded protein response through the upregulation of the ER chaperone GRP78 and caspases as well as evokes $\mathrm{Ca}^{2+}$ flux that induces mitochondrial dysfunction and associated loss of DA neurons (Arduíno et al., 2009; Baek et al.). Interestingly, increased ROS production through PERK/eIF2 $\alpha / A T F 4 / C H O P$ pathway of UPR and concomitant alteration of the mitochondrial network morphology have been reported in PARK20 fibroblasts (Amodio et al.). Emerging evidence supporting significance of altered ER-mitochondria communication suggests that damaged ER-mitochondria signaling could be a potential therapeutic strategy to treat neurodegenerative diseases. 
The present Research Topic is an effort to showcase the significance of MAM in PD pathogenesis. Here, we discuss the recent findings in $\mathrm{PD}$ research with main focus on molecular and cellular mechanisms involving mitochondria and ER. Pathophysiological significance of ER-mitochondria interaction has been demonstrated in the case of PD-related genes, such as $\alpha$-synuclein ( $\alpha$-syn) (Guardia-Laguarta et al., 2014), DJ-1 (Ottolini et al., 2013), PINK1 (Celardo et al., 2016; Gelmetti et al., 2017), and Parkin (Van Laar et al., 2015; Celardo et al., 2016; Gautier et al., 2016; Gelmetti et al., 2017; Zheng et al., 2017). Several clinical cases with diagnosed PD show a welldefined Lewy body pathology (Cookson et al., 2008), which are composed of $\alpha$-syn. Protein aggregation and imbalanced cellular proteostasis are key factors leading to accumulation of misfolded $\alpha$-syn (Lehtonen et al.). Within neurons, $\alpha$-syn is diversely localized to cytosolic and membrane compartments including synaptic vesicles, mitochondria and the ER (GuardiaLaguarta et al., 2015; Colla). Membrane localization of $\alpha$-syn is well-targeted to lipid rafts (detergent-resistant membranes) that are enriched in cholesterol and acidic phospholipids (Fortin et al., 2004). Interestingly, a subpopulation of $\alpha$-syn is shown to be enriched in MAM fraction in immortalized cell lines and in the mouse and human brain (Poston et al., 2013; Guardia-Laguarta et al., 2014; Paillusson et al., 2016). Certainly, identification of the A53T mutation in the gene encoding for $\alpha$-syn (SNCA) provides us better understanding of both the genetics and the neuropathology of PD (Polymeropoulos et al., 1997). It has been demonstrated that A53T mutant showed a decreased association with MAM and an elevated mitochondrial fragmentation, as compared to wild-type $\alpha$-syn (Guardia-Laguarta et al., 2014). Moreover, overexpression of either wild-type or mutant $\alpha$-syn decreases ER-mitochondria contacts (Paillusson et al., 2016). Thus, substantial accumulation of $\alpha$-syn aggregates could be linked to the loss of function of this protein at the MAMs. Interestingly, subcellular localization of $\alpha$-syn to MAM could be related to both normal and pathological states (GuardiaLaguarta et al., 2014, 2015). A recent study demonstrated that $\alpha$-syn binds to VAPB (an ER-mitochondria tethering protein) to disrupt $\mathrm{Ca}^{2+}$ homeostasis and mitochondrial ATP production (Paillusson et al., 2016).

PINK1/Parkin-mediated mitophagy could be an underlying mechanism of nigral DA neuron death in PD (Thomas et al., 2011; Kane et al., 2014; Barodia et al., 2017). ER-mitochondria contact sites were shown to constitute the initiation sites for this process (Yang and Yang, 2013). During mitophagy, PINK1 and BECN1 re-localize at MAM, which induces ER-mitochondria tethering and autophagosome formation (Gelmetti et al., 2017).

\section{REFERENCES}

Abais, J. M., Xia, M., Zhang, Y., Boini, K. M., and Li, P. L. (2015). Redox regulation of NLRP3 inflammasomes: ROS as trigger or effector? Antioxid. Redox Signal. 22, 1111-1129. doi: 10.1089/ars.2014.5994

Arduíno, D. M., Esteves, A. R., Cardoso, S. M., and Oliveira, C. R. (2009). Endoplasmic reticulum and mitochondria interplay mediates apoptotic cell death: relevance to Parkinson's disease.
Parkin expression was significantly increased in the MAM fraction of neurons following glutamate excitotoxicity (Van Laar et al., 2015), which also ubiquitylated several proteins of the ER-mitochondria interface including Mfn2, VDACs and Miro (Sarraf et al., 2013; Pickrell and Youle, 2015). Parkin may regulate ER-mitochondria communication via Mfn2 (Basso et al., 2018). Mitochondrial and ER stress results in an upregulation of Parkin levels via ATF4 (Bouman et al., 2011). ER-mitochondria communication was reported to be increased in fibroblasts from patients with PARK2 or PARK6 mutations compared to control group (Celardo et al., 2016; Gautier et al., 2016). This alteration was associated with higher mitochondrial $\mathrm{Ca}^{2+}$ absorption, upon $\mathrm{IP}_{3} \mathrm{R}$ stimulation. Similar structural changes were observed in MEFs from PARK2 knock-out mice (Gautier et al., 2016). Parkin has recently been reported to co-regulate ERmitochondria communication together with the transcription factor peroxisome proliferator activated receptor $g$ coactivator 1a (PGC-1 $\alpha$ ), a key modulator of mitochondrial biogenesis (Zheng et al., 2017). ER-mitochondria associations have also been linked to the formation of the inflammasome. Cellular stress in neurodegenerative diseases are detected by the innate immune system through pattern recognition receptors (Paillusson et al., 2016). Reactive oxygen species (ROS) from mitochondria are one signal for activation of the NLRP3 inflammasome (Abais et al., 2015). Elevated ROS generation led to NLRP relocation to MAM, which may provide a mechanism whereby NLRP senses damage mitochondria to activate the inflammasome (Zhou et al., 2011). Due to the importance of MAMs in understanding the fundamental mechanisms of PD pathogenesis and their potential use as a therapeutic approach, further research is needed to investigate on the communications between the ER and mitochondria.

\section{AUTHOR CONTRIBUTIONS}

SB collected the relevant references and wrote the manuscript. VT, KP, SK, and VM edited the manuscript and provided thorough reviews on the manuscript.

\section{ACKNOWLEDGMENTS}

We would like to thank you the authors who have contributed to this Research Topic and the dedicated reviewers who helped us reach the highest quality standards. We gratefully acknowledge the valuable input of the Frontiers editorial team members for their support in editing and publishing the scientific content. 
Bouman, L., Schlierf, A., Lutz, A. K., Shan, J., Deinlein, A., Kast, J., et al. (2011). Parkin is transcriptionally regulated by ATF4: evidence for an interconnection between mitochondrial stress and ER stress. Cell Death Differ. 18, 769-782. doi: 10.1038/cdd.2 010.142

Celardo, I., Costa, A. C., Lehmann, S., Jones, C., Wood, N., Mencacci, N. E., et al. (2016). Mitofusin-mediated ER stress triggers neurodegeneration in pink1/parkin models of Parkinson's disease. Cell Death Dis. 7:e2271. doi: $10.1038 /$ cddis. 2016.173

Cookson, M. R., Hardy, J., and Lewis, P. A. (2008). Genetic neuropathology of Parkinson's disease. Int. J. Clin. Exp. Pathol. 1, 217-231.

Erpapazoglou, Z., Mouton-Liger, F., and Corti, O. (2017). From dysfunctional endoplasmic reticulum-mitochondria coupling to neurodegeneration. Neurochem. Int. 109, 171-183. doi: 10.1016/j.neuint.2017. 03.021

Fortin, D. L., Troyer, M. D., Nakamura, K., Kubo, S., Anthony, M. D., and Edwards, R. H. (2004). Lipid rafts mediate the synaptic localization of alpha-synuclein. J. Neurosci. 24, 6715-6723. doi: 10.1523/JNEUROSCI.1594-04.2004

Gautier, C. A., Erpapazoglou, Z., Mouton-Liger, F., Muriel, M. P., Cormier, F., Bigou, S., et al. (2016). The endoplasmic reticulum-mitochondria interface is perturbed in PARK2 knockout mice and patients with PARK2 mutations. Hum. Mol. Genet. 25, 2972-2984. doi: 10.1093/hmg/ddw148

Gelmetti, V., De Rosa, P., Torosantucci, L., Marini, E. S., Romagnoli, A., Di Rienzo, M., et al. (2017). PINK1 and BECN1 relocalize at mitochondria-associated membranes during mitophagy and promote ERmitochondria tethering and autophagosome formation. Autophagy 13, 654-669. doi: 10.1080/15548627.2016.1277309

Gómez-Suaga, P., Bravo-San Pedro, J. M., González-Polo, R. A., Fuentes, J. M., and Niso-Santano, M. (2018). ER-mitochondria signaling in Parkinson's disease. Cell Death Dis. 9:337. doi: 10.1038/s41419-017-0079-3

Guardia-Laguarta, C., Area-Gomez, E., Rüb, C., Liu, Y., Magran,é, J., Becker, D., et al. (2014). alpha-Synuclein is localized to mitochondria-associated ER membranes. J. Neurosci. 34, 249-259. doi: 10.1523/JNEUROSCI.2507-13.2014

Guardia-Laguarta, C., Area-Gomez, E., Schon, E. A., and Przedborski, S. (2015). Novel subcellular localization for alpha-synuclein: possible functional consequences. Front. Neuroanat. 9:17. doi: 10.3389/fnana.2015.00017

Kane, L. A., Lazarou, M., Fogel, A. I., Li, Y., Yamano, K., Sarraf, S. A., et al. (2014). PINK1 phosphorylates ubiquitin to activate Parkin E3 ubiquitin ligase activity. J. Cell Biol. 205, 143-153. doi: 10.1083/jcb.201 402104

Krols, M., van Isterdael, G., Asselbergh, B., Kremer, A., Lippens, S., Timmerman, V., et al. (2016). Mitochondria-associated membranes as hubs for neurodegeneration. Acta Neuropathol. 131, 505-523. doi: 10.1007/s00401-015-1528-7

Lee, K. S., Huh, S., Lee, S., Wu, Z., Kim, A. K., Kang, H. Y., Lu, B., et al. (2018). Altered ER-mitochondria contact impacts mitochondria calcium homeostasis and contributes to neurodegeneration in vivo in disease models. Proc. Natl. Acad. Sci. U.S.A. 115, E8844-E8853. doi: 10.1073/pnas.17211 36115

Ottolini, D., Cal,ì, T., Negro, A., and Brini, M. (2013). The Parkinson diseaserelated protein DJ-1 counteracts mitochondrial impairment induced by the tumour suppressor protein p53 by enhancing endoplasmic reticulum-mitochondria tethering. Hum. Mol. Genet. 22, 2152-2168. doi: $10.1093 / \mathrm{hmg} / \mathrm{ddt} 068$
Paillusson, S., Stoica, R., Gomez-Suaga, P., Lau, D. H. W., Mueller, S., Miller, T., et al. (2016). There's something wrong with my MAM; the ER-mitochondria axis and neurodegenerative diseases. Trends Neurosci. 39, 146-157. doi: 10.1016/j.tins.2016.01.008

Pickrell, A. M., and Youle, R. J. (2015). The roles of PINK1, parkin, and mitochondrial fidelity in Parkinson's disease. Neuron 85, 257-273. doi: 10.1016/j.neuron.2014.12.007

Polymeropoulos, M. H., Lavedan, C., Leroy, E., Ide, S. E., Dehejia, A., Dutra, A., et al. (1997). Mutation in the alpha-synuclein gene identified in families with Parkinson's disease. Science 276, 2045-2047. doi: $10.1126 /$ science.276.5321.2045

Poston, C. N., Krishnan, S. C., and Bazemore-Walker, C. R. (2013). Indepth proteomic analysis of mammalian mitochondria-associated membranes (MAM). J. Proteomics 79, 219-230. doi: 10.1016/j.jprot.2012.12.018

Rodríguez-Arribas, M., Yakhine-Diop, S. M. S., Pedro, J. M. B., Gómez-Suaga, P., Gómez-Sánchez, R., Martínez-Chacón, G., et al. (2017). MitochondriaAssociated Membranes (MAMs): Overview and Its Role in Parkinson's Disease. Mol. Neurobiol. 54, 6287-6303. doi: 10.1007/s12035-016-0140-8

Sarraf, S. A., Raman, M., Guarani-Pereira, V., Sowa, M. E., Huttlin, E. L., Gygi, S. P., et al. (2013). Landscape of the PARKIN-dependent ubiquitylome in response to mitochondrial depolarization. Nature 496, 372-376. doi: 10.1038/nature12043

Thomas, K. J., McCoy, M. K., Blackinton, J., Beilina, A., van der Brug, M., Sandebring, A., et al. (2011). DJ-1 acts in parallel to the PINK1/parkin pathway to control mitochondrial function and autophagy. Hum. Mol. Genet. 20, 40-50. doi: $10.1093 / \mathrm{hmg} / \mathrm{ddq} 430$

Valadas, J. S., Esposito, G., Vandekerkhove, D., Miskiewicz, K., Deaulmerie, L., Raitano, S., et al. (2018). ER lipid defects in neuropeptidergic neurons impair sleep patterns in Parkinson's disease. Neuron 98, 1155-1169 e1156. doi: 10.1016/j.neuron.2018.05.022

Van Laar, V. S., Roy, N., Liu, A., Rajprohat, S., Arnold, B., Dukes, A. A., et al. (2015). Glutamate excitotoxicity in neurons triggers mitochondrial and endoplasmic reticulum accumulation of Parkin, and, in the presence of $\mathrm{N}$-acetyl cysteine, mitophagy. Neurobiol. Dis. 74, 180-193. doi: 10.1016/j.nbd.2014.11.015

Yang, J. Y., and Yang, W. Y. (2013). Bit-by-bit autophagic removal of parkinlabelled mitochondria. Nat. Commun. 4:2428. doi: 10.1038/ncomms3428

Zheng, L., Bernard-Marissal, N., Moullan, N., D’Amico, D., Auwerx, J., Moore, D. J., et al. (2017). Parkin functionally interacts with PGC-1alpha to preserve mitochondria and protect dopaminergic neurons. Hum. Mol. Genet. 26, 582-598. doi: 10.1093/hmg/ddw418

Zhou, R., Yazdi, A. S., Menu, P., and Tschopp, J. (2011). A role for mitochondria in NLRP3 inflammasome activation. Nature 469, 221-225. doi: $10.1038 /$ nature09663

Conflict of Interest: The authors declare that the research was conducted in the absence of any commercial or financial relationships that could be construed as a potential conflict of interest.

Copyright (c) 2019 Barodia, Prabhakaran, Karunakaran, Mishra and Tapias. This is an open-access article distributed under the terms of the Creative Commons Attribution License (CC BY). The use, distribution or reproduction in other forums is permitted, provided the original author(s) and the copyright owner(s) are credited and that the original publication in this journal is cited, in accordance with accepted academic practice. No use, distribution or reproduction is permitted which does not comply with these terms. 